\title{
CW LASER OSCILLATIONS IN Cd II IN AN ELECTRON BEAM CREATED PLASMA
}

\author{
J.J. ROCCA ${ }^{1}$, J.D. MEYER and G.J. COLLINS ${ }^{2}$ \\ Department of Electrical Engineering, Colorado State University, Fort Collins, CO 80523, USA
}

Received 11 January 1982

Revised manuscript received 25 May 1982

A cw Cd II laser has been demonstrated using electron beam excitation. Cw laser oscillation has been obtained on six transitions of $\mathrm{Cd} \mathrm{II}$ in a $\mathrm{He}-\mathrm{Cd}$ plasma. Output power as a function of electron beam discharge parameters has been investigated.

The green laser lines of Cd II fail to oscillate cw in a positive column discharge. $\mathrm{Cw}$ laser oscillation on the $5337 \AA\left(4 \mathrm{f}^{2} \mathrm{~F}_{5 / 2}^{\mathrm{o}} \rightarrow 5 \mathrm{~d}^{2} \mathrm{D}_{3 / 2}\right)$ and $5378 \AA\left(4 \mathrm{f}^{2} \mathrm{~F}_{7 / 2}^{\mathrm{o}}\right.$ $\rightarrow 5 \mathrm{~d}^{2} \mathrm{D}_{5 / 2}$ ) transitions of singly ionized cadmium was first obtained by Schuebel [1] in a slotted hollow cathode discharge. Recently, we proposed the use of a dc electron beam to excite cw ion lasers [2]. Here we report $\mathrm{cw}$ laser oscillation on these green transitions as well as on the $4416,6355,6360$ and $8067 \AA$ lines of Cd II, obtained using a dc electron beam discharge as the laser active medium.

The electron beam created plasma presents a nonmaxwellian electron energy distribution that has high density of energetic electrons, hence, this plasma is a new and attractive active medium for ion lasers. As a specific example, consider the $\mathrm{He}-\mathrm{Cd}$ laser. In positive column $\mathrm{He}-\mathrm{Cd}$ discharges, the electron temperature decreases very rapidly with increasing cadmium pressure [3]. As a result, the metal vapor concentration and the electron temperature cannot be independently optimized. In the electron beam excitation scheme, the Cd concentration in the plasma tube and the parameters of the electron beam discharge can be more independently varied.

The experimental setup used to obtain cw laser oscillation on Cd II transitions is shown in fig. 1. We

\footnotetext{
1 Spectra Physics Industrial Fellow.

2 Alfred Sloan Fellow, 1979-82.
}

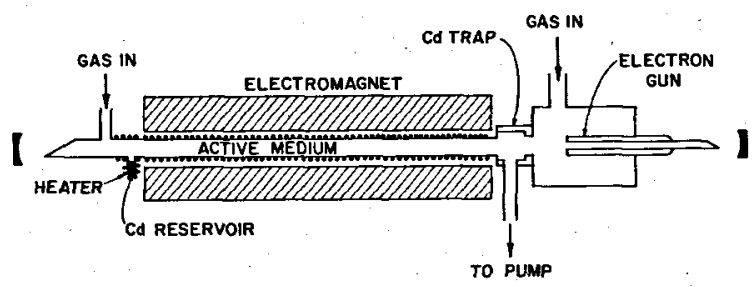

Fig. 1. Schematic diagram of the electron beam $\mathrm{Cd}$ ion laser.

have used a dc electron beam of energy between 1 and $5 \mathrm{keV}$ to excite a $\mathrm{He}-\mathrm{Cd}$ gas mixture. The electron beam is generated by a glow discharge electron gun [4] that provides an optical path throughout the axis. This permits us to match the electron beam created plasma volume with the corresponding volume of the optical resonator. Electron beam discharge currents up to $1 \mathrm{~A}$ are generated by the glow discharge electron gun. This electron gun operates in helium at pressures between 0.1 and 3 Torr without differential pumping. The electron gun is mounted in a micropositioner that permits one to accurately and easily align it with respect to the axis of the plasma tube. The stainless steel plasma tube is $1.1 \mathrm{~cm}$ in diameter and $100 \mathrm{~cm}$ long. The electron gun chamber and the plasma tube are separated by a water-cooled metal vapor trap that is connected to the vacuum pump (see fig. 1). At the opposite end of the plasma tube there is a cadmium source reservoir. The plasma tube is surrounded by 
an oven that provides a temperature higher than the one corresponding to the independently heated $\mathrm{Cd}$ reservoir. In this way we avoid condensation of $\mathbf{C d}$ on the walls of the plasma tube. A good distribution of the $\mathrm{Cd}$ vapor in the plasma region is achieved by flowing He at 200 and 400 standard cc per minute. The axis of the plasma tube is coincident with the axis of a solenoid capable of producing a magnetic field of up to $4.2 \mathrm{kG}$. The axial magnetic field helps to efficiently deposit the electron beam energy in the plasma, reducing collisions of energetic beam electrons with the walls.

The metal vapor trap used in the first laser experiments had a circular opening of $0.6 \mathrm{~cm}$ in diameter connecting the electron gun chamber and the plasma tube. This trap was stopping part of the electron beam from reaching the active medium in the plasma tube. In this first experiment we obtained laser action on the 5337, 5378 and $8067 \AA$ transitions of Cd II. All three laser lines had a current threshold between 60 and $70 \mathrm{~mA}$, corresponding to a discharge voltage of $1.3 \mathrm{kV}$. Fig. 2 shows the laser power output as a function of the average helium pressure in the plasma tube at a cadmium reservoir temperature of $300^{\circ} \mathrm{C}$ and an electron beam discharge current of $700 \mathrm{~mA}$. Fig. 3 shows the laser output power as a function of electron

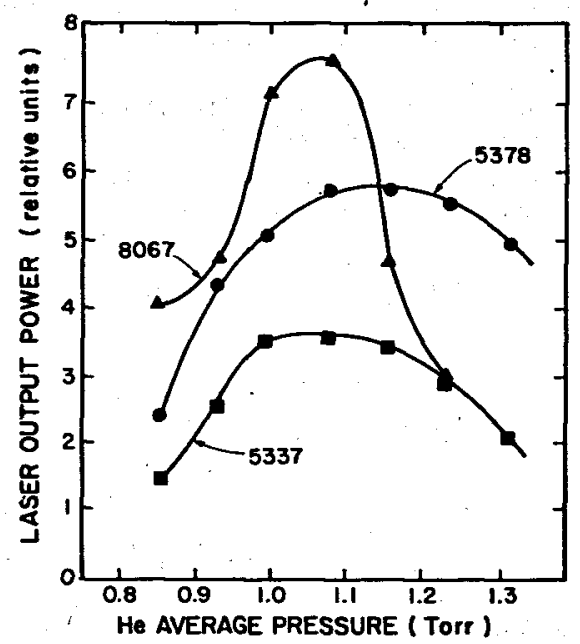

Fig. 2. Laser output power as a function of the average helium pressure in the plasma tube at a current of $700 \mathrm{~mA}$ and a $\mathrm{Cd}$ reservoir temperature of $300^{\circ} \mathrm{C}$. Magnetic field $3.9 \mathrm{kG}$. Wavelengths are given in $A$ and the intensity of the $5337 \AA$ line is $2 X$ its actual value.

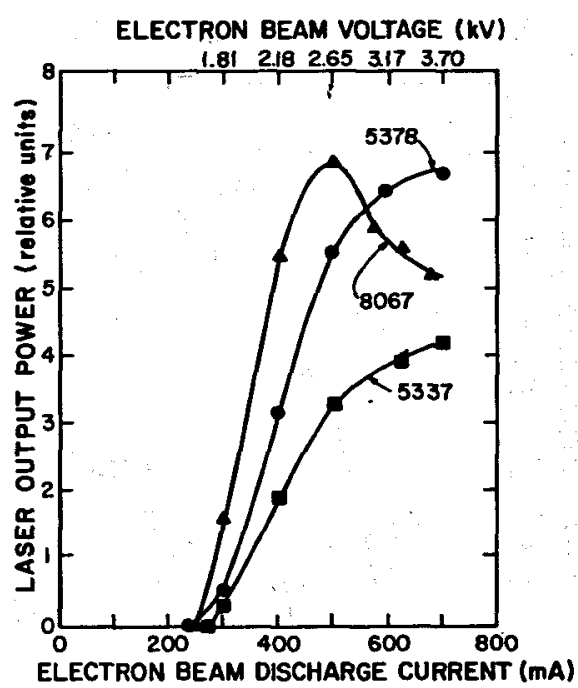

Fig. 3. Laser output power as a function of electron beam discharge current and voltage. Cd reservoir temperature $300^{\circ} \mathrm{C}$. Magnetic field $3.9 \mathrm{kG}$. Average helium pressure in the plasma tube 1.15 Torr. Wavelengths are given in $A$ and the relative intensity of the $5337 \AA$ line is $2 \times$ ist actual value.

beam discharge current and voltage for a magnetic field of $3.9 \mathrm{kG}$, a Cd reservoir temperature of $300^{\circ} \mathrm{C}$, and an average helium pressure in the plasma tube of 1.15 Torr. Under this condition the laser output power of the green lines continues to rise up to the maximum current, but the output laser power of the $8067 \AA$ line saturates and decreases as the electron beam current is increased. This saturation is less evident at weaker magnetic field strengths.

In later experiments we redesigned the metal vapor trap allowing a better deposition of the electron beam into the $\mathrm{He}-\mathrm{Cd}$ mixture. Enlarging the opening of the metal vapor trap to $1.0 \mathrm{~cm}$ in diameter, laser action was obtained on three additional transitions of Cd II: 4416, 6360 and $6355 \AA$. Also, the optimum magnetic field value for optimum laser output dropped significantly. The laser output power of the red lines as a function of discharge current and voltage is shown in fig. 4 for an optimum magnetic field of $1.25 \mathrm{kG}$.

The most intense laser lines were the green lines. Using an unoptimized optical resonator with an output coupler reflectivity of $95.5 \%$ at $5350 \AA$ a maximum laser power of $80 \mathrm{~mW}$ was obtained for the combination of the 5378 and $5337 \AA$ transitions. The output power of the other laser transitions was not optimized or measured. 


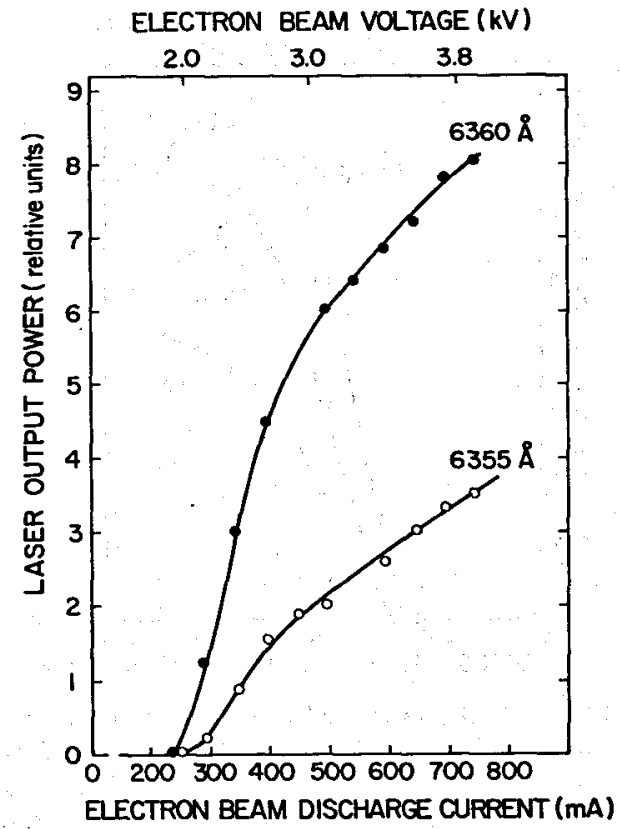

Fig. 4. Laser output power of the $6355 \AA$ and $6360 \AA$ as a function of electron beam discharge current and voltage. $\mathrm{Cd}$ reservoir temperature $315^{\circ} \mathrm{C}$, magnetic field $1.25 \mathrm{kG}$. Average helium pressure in the plasma tube 2.5 Torr.

In hollow cathode discharges, the dominant excitation mechanism for the transitions at 5378 and $5337 \AA$ is suggested to be radiative cascade to the upper laser levels via the 6360 and $6355 \AA$ transitions respectively [1]. These two red transitions are laser lines that are populated by charge transfer collisions between $\mathrm{He}$ ions and ground state $\mathrm{Cd}$ atoms. To examine the possibility of direct electron impact excitation of the green Cd II transitions from the Cd I ground state, we replaced He as a buffer gas. First, laser radiation in a pure $\mathrm{He}-\mathrm{Cd}$ mixture was established. Then, we observed the laser output power decreased as a function of increasing percentage of $\mathrm{Ne}$ until it has been completely quenched with only a 1: $3 \mathrm{Ne}$ to He ratio. This suggests that the charge transfer process involving $\mathrm{He}^{+}$ and ground state $\mathrm{Cd}$ followed by radiative cascade makes the dominant contribution to the population of the $4 \mathrm{f}^{2} \mathrm{~F}$ Cd II laser levels.

In summary we have obtained $\mathrm{cw}$ laser radiation for the first time in singly ionized cadmium using electron beam excitation of a $\mathrm{He}-\mathrm{Cd}$ gas mixture. $\mathrm{A} \mathrm{cw}$ laser output power of $80 \mathrm{~mW}$ was obtained for the combination of the green Cd II laser transitions. The use of a dc electron beam presents a new method of exciting ion lasers.

The authors wish to thank T. Burnell for skilled technical assistance. This work was supported by the National Science Foundation.

\section{References}

[1] W.K. Schuebel, Appl. Phys. Lett. 16 (1970) 470.

[2] J.J. Rocca, J.D. Meyer, A. Yu and G.J. Collins, Thirty fourth gaseous electronic conference, Boston, October 1981.

[3] T. Goto, A. Kawahara, G.J. Collins and S. Hattori, J. Appl. Phys. 42 (1971) 3816.

[4] J.J. Rocca, J.D. Meyer and G.J. Collins, Phys. Lett. 87A (1982) 237. 